\title{
Massive stars: flare activity due to infalls of comet-like bodies
}

\author{
Subhon Ibadov ${ }^{1,2}$ and Firuz S. Ibodov ${ }^{1}$ \\ ${ }^{1}$ Lomonosov Moscow State University, SAI, Moscow, Russia \\ email: ibadovsu@yandex.ru \\ ${ }^{2}$ Institute of Astrophysics, TAS, Dushanbe, Tajikistan
}

\begin{abstract}
Passages of comet-like bodies through the atmosphere/chromosphere of massive stars at velocities more than $600 \mathrm{~km} / \mathrm{s}$ will be accompanied, due to aerodynamic effects as crushing and flattening, by impulse generation of hot plasma within a relatively very thin layer near the stellar surface/photosphere as well as "blast" shock wave, i.e., impact-generated photospheric stellar/solar flares. Observational manifestations of such high-temperature phenomena will be eruption of the explosive layer's hot plasma, on materials of the star and "exploding" comet nuclei, into the circumstellar environment and variable anomalies in chemical abundances of metal atoms/ions like Fe, Si etc. Interferometric and spectroscopic observations/monitoring of young massive stars with dense protoplanetary discs are of interest for massive stars physics/evolution, including identification of mechanisms for massive stars variability.
\end{abstract}

Keywords. massive stars, impact-generated stellar flares, comet-like bodies

\section{Introduction}

High-resolution spectroscopic observations of young stars with dense protoplanetary discs like $\beta$ Pictoris as well as coronagraphic observations by solar missions indicate the presence of fluxes of comet-like evaporating bodies (FEBs), falling onto Sun/stars (see, e.g. Lagrange et al. 1987; Beust et al. 1996, http://sungrazer.nrl.navy.mil/).

Disintegration of nuclei of sungrazing comets considered in a vacuum leads to a decrease in their radii to less than 10-20 m prior to impact (Weissman 1983).

Meanwhile, comet-like bodies fall onto massive stars with high orbital velocities $V_{*} \geqslant$ $V_{\odot}\left(V_{\odot}=617 \mathrm{~km} / \mathrm{s}\right.$ is the parabolic velocity for the Sun $)$. This indicates the possibility of high energy processes in comets (cf. Ibadov 1990, 1996; Ibadov et al. 2009; Ibodov et al. 2010; Ibodov \& Ibadov 2011, 2014). Impulse release of a large energy, up to $10^{32} \mathrm{erg}$, is possible due to the magnetic reconnection in the active Sun (cf. Somov 1992; Walder et al. 2012). We consider physical processes accompanying the passage of star-impacting comet nuclei through the stellar atmosphere, i.e., processes that are capable to lead to massive stars flare activity.

\section{Massive stars comet-induced flares}

To find, analytically, the law for the rate of the loss of kinetic energy of aerodynamically fully crushed and transversally expanding comet-like body along the height of the star atmosphere, $h$, we modify the basic equations of the physical theory of meteors (aerodynamic deceleration/evaporation) using the parameter $r=r(h)=\left\{\exp \left[\left(h_{*}-h\right) / H\right]\right\}-1$. We use also the comet orbital velocity near the star surface, $V_{*}=V_{\odot}\left(M_{*} R_{\odot} / M_{\odot} R_{*}\right)^{1 / 2}$. Here $M_{*}, M_{\odot}$ are the masses of the star and the Sun, $R_{*}, R_{\odot}$ are their radii; $h_{*}$ is the 
onset height for aerodynamic crushing of comet nucleus, $H$ is the atmosphere height scale.

The energy released around the height of $h=h_{\mathrm{m}}$ (the height of the maximum rate of loss of kinetic energy of the crushed comet nucleus), manifesting as "explosion" in the atmosphere around $r=r_{\mathrm{m}}=r_{\mathrm{e}}$, and the initial temperature of plasma produced within the decelerating/exploding layer with the width, $\Delta h_{\mathrm{d}}=\Delta h_{\mathrm{e}} \approx 0.7 \mathrm{H}$, are

$$
E_{\mathrm{e}}=\frac{\pi \rho_{\mathrm{n}} R_{\mathrm{n}}^{3} V_{*}^{2}}{3 e}, \quad T_{0}=\frac{A m_{\mathrm{p}} V_{*}^{2}}{12 e k\left(1+z+2 x_{1} / 3\right)},
$$

where $\rho_{\mathrm{n}}, R_{\mathrm{n}}$ are the nucleus density and initial radius, $A$ is the mean atomic number, $m_{\mathrm{p}}$ is the proton mass, $k$ is the Boltzmann constant, $z$ is the mean charge multiplicity of plasma ions, $x_{1}$ is the mean relative ionization potential; the theory was tested using for the 2013 Chelyabinsk superbolide explosion (cf. Ibadov et al. 2009; Ibadov 2012; Ibodov \& Ibadov 2011; Grigoryan et al. 2013).

Using (2.1) with $\rho_{\mathrm{n}}=0.5 \mathrm{~g} / \mathrm{cm}^{3}, R_{\mathrm{n}}=10^{5} \mathrm{~cm}, V_{*}=V_{0}, A=20, z=5, x_{1}=3$ we have $\Delta h_{\mathrm{e}}=140 \mathrm{~km}, E_{\mathrm{e}}=7 \cdot 10^{29} \mathrm{erg}, T_{0}=7 \cdot 10^{6} \mathrm{~K}$. Hence, "superflares" may be due to impacts with comets like comet Hale-Bopp 1995 OI (cf. Ibadov et al. 1999; Grigoryan et al. 2000; Ibadov et al. 2009; Eichler \& Mordecai 2012; Ibodov \& Ibadov 2014).

\section{Conclusions}

Impacts of sufficiently large, 100-meter or more, comet-like bodies with stars like the Sun and/or more massive ones will be accompanied by impulse production of a hightemperature plasma, strong "blast" shock wave, shock wave induced ejection of ionized photospheric and cometary matter.

Anomalously intense variable emissions of metal atoms and ions produced during the nucleus "explosion" near the stellar/solar surface may serve as possible indicators of comet impact-generated photospheric stellar/solar flares.

\section{Acknowledgements}

The authors are grateful to Prof. G. Meynet for invitation, Dr. G.M. Rudnitskij for useful discussions and V. Vybornov for technical help. The hospitality of the IAUS 307 LOC and MSU is sincerely acknowledged.

\section{References}

Beust, H., Lagrange, A.-M., Plazy, F., \& Mouillet, D. 1996, A\&SA 310, 181

Eichler, D. \& Mordecai, D. 2012, ApJ (Letters) 761, L27

Grigoryan, S., Ibadov, S., \& Ibodov, F. 2000, Doklady Physics 45(9), 463

Grigoryan, S. S., Ibodov, F. S., \& Ibadov, S. I. 2013, Solar System Research 47, 268

Ibadov, S. 1990, Icarus 86, 283

Ibadov, S. 1996, Physical processes in comets and related objects

Ibadov, S. 2012, in IAU Symposium, Vol. 283 of IAU Symposium, pp 392-393

Ibadov, S., Ibodov, F. S., \& Grigorian, S. S. 1999, Romanian Astronomical Journal 9, 125

Ibadov, S., Ibodov, F. S., \& Grigorian, S. S. 2009, in N. Gopalswamy \& D. F. Webb (eds.), IAU Symposium, Vol. 257 of IAU Symposium, pp 341-343

Ibodov, F. S., Grigorian, S. S., \& Ibadov, S. 2010, in J. A. Fernandez, D. Lazzaro, D. Prialnik, \& R. Schulz (eds.), IAU Symposium, Vol. 263 of IAU Symposium, pp 269-271

Ibodov, F. S. \& Ibadov, S. 2011, in A. Bonanno, E. de Gouveia Dal Pino, \& A. G. Kosovichev (eds.), IAU Symposium, Vol. 274 of IAU Symposium, pp 92-94 
Ibodov, F. S. \& Ibadov, S. 2014, in B. Schmieder, J.-M. Malherbe, \& S. T. Wu (eds.), IAU Symposium, Vol. 300 of IAU Symposium, pp 509-511

Lagrange, A. M., Ferlet, R., \& Vidal-Madjar, A. 1987, A\&\& 173, 289

Somov, B. V. (ed.) 1992, Physical processes in solar flares., Vol. 172 of Astrophysics and Space Science Library

Walder, R., Folini, D., \& Meynet, G. 2012, Space Sci. Revs 166, 145

Weissman, P. R. 1983, Icarus 55, 448

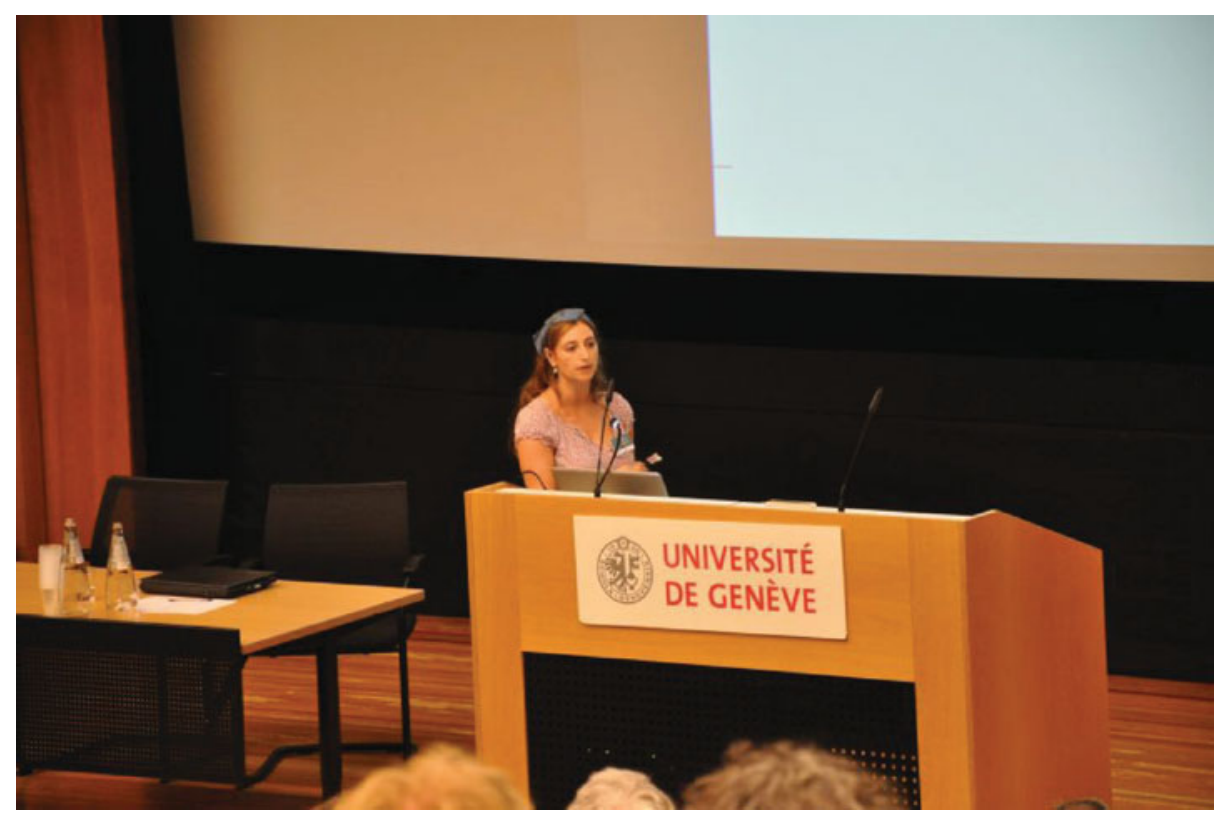

Melina Bersten

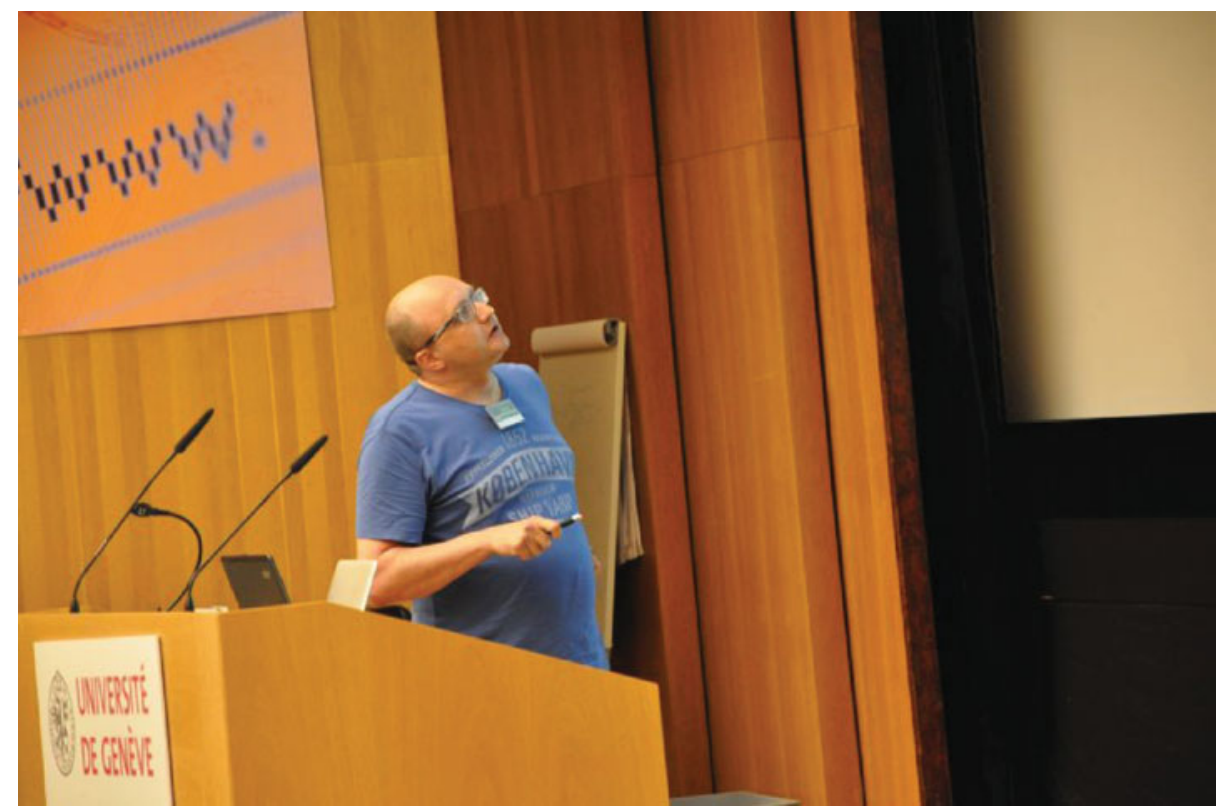

Götz Gräfener 